\title{
An Adaptive Energy Aware Algorithm for Reliable Transmission Sensor Networks
}

\author{
Shi-Hua Cao ${ }^{1}$, Qi-Hui Wang ${ }^{1}$ and Li-Dong Wang ${ }^{1}$ \\ 1. Qianjiang College, Hangzhou Normal University, \\ Hangzhou, 310036, China \\ caoshihua@126.com
}

\begin{abstract}
Route maintenance and repair process plays an important role in mobile selforganizing network. On the basis of considering route stability and energy aware routing protocol, a new scheme is proposed in this paper. Firstly, signal strength, rest energy and port queue length are periodically and alternately detected; secondly, cross-layer method is adopted for route maintenance, and the alternate route is actively established for data transmission according to the routing repair mechanism "Make-before-Break"; finally, relevant experiment is carried out in NS2 to simulate and compare the proposed protocol and other similar routing protocols such as RSEA and LAER. The experiment result shows that the new scheme has good performance in the aspects of data packet delivery ratio, overhead control and end-to-end delay.
\end{abstract}

Keywords: Signal strength; Sensor network; Stability; Energy aware; Routing

\section{Introduction}

As a set composed of some wireless mobile devices (also known as nodes) within a certain range, the mobile self-organizing network can realize mutual communication, self-configuration and maintenance, without the assistance of any other fixed infrastructure for supporting and management. Due to the features of dynamic topology, limited bandwidth, battery and CPU resources, multi-hop communication, etc., such network also brings special challenges to routing protocol design [1].

Predecessors have proposed several routing protocols for MANNET, and these routing protocols are divided into proactive routing protocols and reactive routing protocols according to the route discovery principle. Specifically, the reactive ondemand routing protocol employs PREQ-RREP process to determine the route only when needing to transmit a certain data packet. Most routing protocols employ minimum hop count as the routing measurement. Predecessors found that the shortest path, especially the shortest path in high-density self-organizing network, had short life, even though low mobility was caused by edge effect [2]. However, these routing protocols all fail to solve the problem about how to reduce the link interruption during data transmission process. In most on-demand routing algorithms, certain time shall be taken to detect link faults and then the routing recovery process shall be started [3, 4]. In this way, many resources, such as bandwidth and energy, will be consumed and additional time delay will be also introduced. Meanwhile, the network efficiency will be significantly reduced due to new route discovery. Specifically, in literature [5], link stability is defined as the measurement for link stability degree and communication duration, and signal strength is a parameter used for evaluating link stability. In literature [6], crosslayer method and node mobility prediction are proposed therein to improve the routing protocol so as to realize the stable routing based on cross-layer coordination 
and node position prediction. In literature [7], the graph theory method is proposed to realize network reconnection and further realize stable routing. Although these protocols can inordinately realize stable routing, yet they have their own applicable scopes. In literature [8], link stability and route stability are calculated by N.Sharma according to the received signal strength so as to propose RSQR, and the links are divided into stable links and instable links according to the threshold value. EBL is proposed in literature [9], and the author regards link stability and remaining battery capacity as the very important parts, wherein EBL can not only improve energy utilization, but also reduce network division. LAER is proposed in literature [10] to consider link stability and energy wastage rate jointly for the route discovery, thus to reduce control overhead and balance traffic load. PERRA as a reactive routing protocol is proposed in literature [11] to consider link stability and energy efficiency at the same time. Due to the constraint to the maintenance of congestion and alternate routes, the control overhead is significantly reduced. Meanwhile, the expected route life is roughly predicted according to the parameters of node battery capacity and link stability, thus indicating that it is more suitable to select stable links.

A novel "Make-before-Break" mechanism is proposed in this paper to strengthen the route maintenance in RSEA- AODV. In case of the existence of any link interruption cause such as mobility, energy consumption and congestion, the crosslayer method can be adopted to find an alternate route for data transmission. The simulation result shows that in high-load and high-dynamic environment, the performance of the proposed method is superior to those of RSEA protocol and LAER protocol. By virtue of backup routing, in case of unpredictable link interruption, AODV-BR [12] can realize rapid routing recovery, and the routing can be updated especially through data packet and RREP packet monitoring. However, high control information overhead and collision will be caused in high-density network.

\section{Problem description}

The topological structure of MANET can be expressed as undirected graph $G=(V, E)$, wherein $V$ is the node set and $\mathrm{E}$ is the node connection edge set. For $P(u, v)=\left\{P_{0}, P_{1}, P_{2}, \ldots, P_{n}\right\}$, each $P_{i}$ is a feasible route between $\mathrm{u}$ and $\mathrm{v}$. In consideration of route stability and rest energy, the problem regarding the optimal route from the source to the destination can be described as follows:

$$
\begin{aligned}
& f 1=\prod_{e \in P_{i}} L S(e) \\
& f 2=\prod_{e \in P_{i}} C e(t)
\end{aligned}
$$

Therein,

$$
C e(t)=\left(\frac{R_{i}}{F_{i}}\right)
$$

Meanwhile, $R_{i}>E T h r 1$ and $q_{i}<Q T h r e s h$ are true.

In the above formula, $R_{i}$ and $F_{i}$ respectively refer to the rest energy and the total energy of the node.

Importance factors $w 1$ and $w 2$ are provided to each index of the target, and the above optimization problem can be converted into a single target problem, as shown in the following formula: 


$$
\begin{aligned}
& \operatorname{RFact}\left(P_{i}\right)=w 1 \cdot f 1+w 2 \cdot f 2 \\
& =w 1 \cdot \prod_{e \in P_{i}} L S(e)+w 2 \prod_{e \in P_{i}} C e(t)
\end{aligned}
$$

In the above formula, $p 1+p 2=1$ is true.

The sum of the maximum target is expressed as follows:

$$
\operatorname{RFact}\left(P_{i}\right)=\max \left\{\operatorname{RFact}\left(P_{1}\right), \operatorname{RFact}\left(P_{2}\right), \ldots, \operatorname{RFact}\left(P_{n}\right)\right\}
$$

\section{The Proposed Improved Routing Protocol}

The specific steps of RSEA-RM (Route Stability and Energy Aware Routing with Route Maintenance) routing protocol are mainly discussed in this section, and mainly include route discovery, routing and route maintenance.

\subsection{Route Discovery}

When needing to send data packet to a certain destination node, the node concerned will search the routes in the route cache. If the route leading to the destination node $\mathrm{D}$ is unavailable, then the source node $\mathrm{S}$ will send the routing request RREQ to the neighbor node. After receiving RREQ data packet, the intermediate node will detect the strength thereof, thus to receive the data packet and the rest energy of the node. If the signal strength and the rest energy exceed the corresponding threshold values, the node will check the load index thereof; or else, it will abandon the routing request. If the queue length exceeds QThresh value, then the routing request data packet will be lost in order to avoid the subsequent data transmission congestion. If the signal strength exceeds SThrl, then LS shall be set as 1. If the signal strength is between SThr1 and SThr2, then LS shall be calculated and obtained according to DSS (Differentiated Signal Strength).

Before the calculated values are sent to the neighbor node, APST (Accumulated Path Stability) and AE (Accumulated Energy) calculation function is adopted to update the head segment of RREQ data packet. The calculated stability value will be also updated in the neighbor information sheet. Afterwards, the routing request will carry updated APST and AE values and forward them to the neighbor node thereof.

\subsection{Routing}

When receiving the first RREQ, the destination node will enable the timer $\Delta t$ for $\mathrm{t}$ seconds. According to Objective Function (5), received APST and AEC values in the routing request will be adopted to calculate route reliability. The destination node has stored all RREQs of the reliability values in the routing cache. After timer expires, the destination node will find the route with minimum target value and then send RREP thereto. After timer $\Delta t$ expires, the arriving routing request will be abandoned.

\section{Algorithm 1. Target Node Execution}

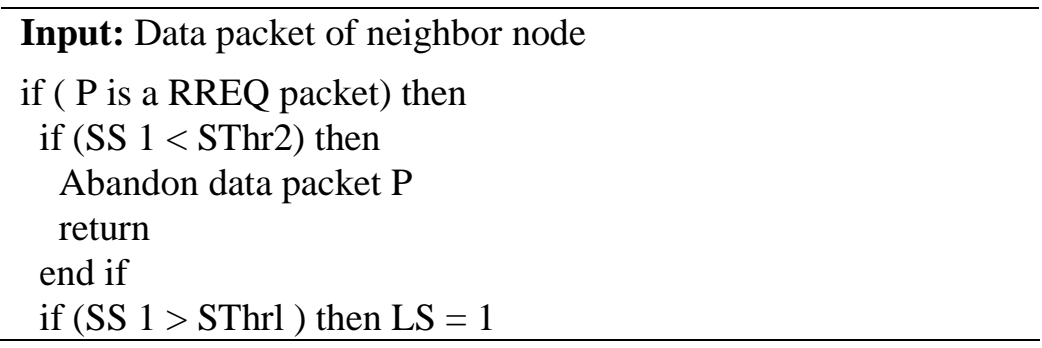




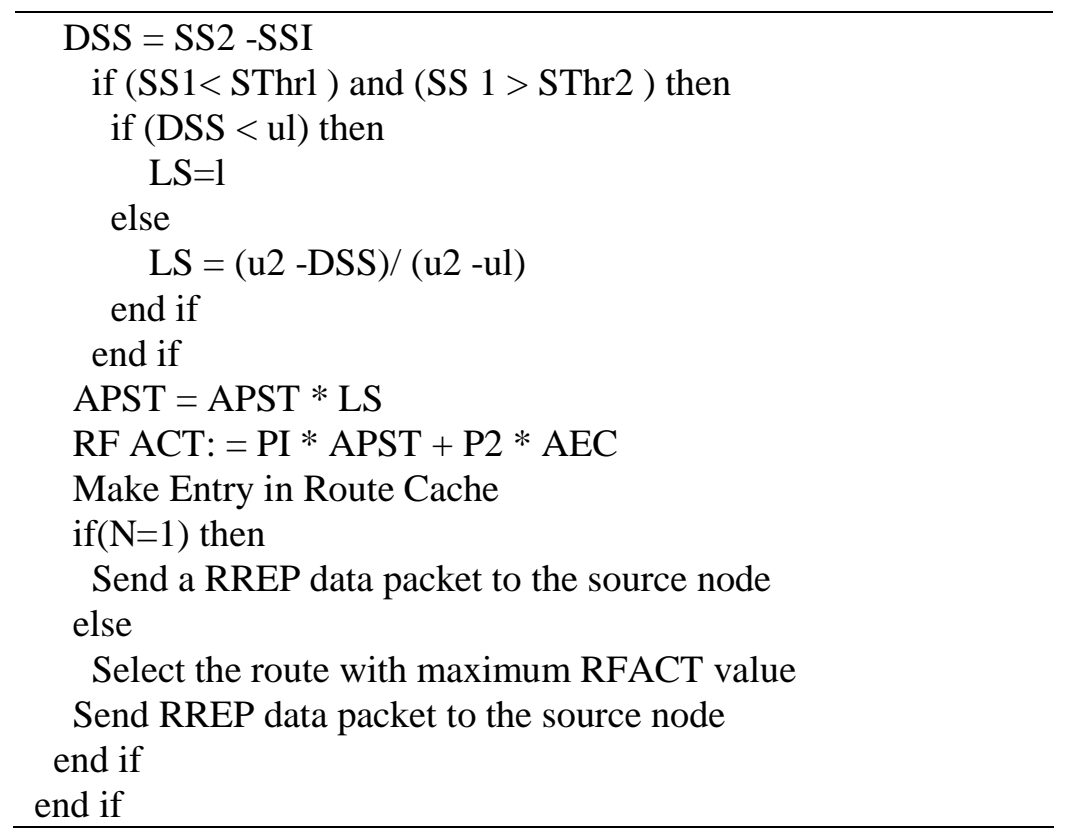

\subsection{Route Maintenance}

"Make-before-Break" mechanism as shown in Algorithm 2 can rapidly adapt to the link interruption caused by mobility, energy loss and congestion. This mechanism is executed every $\mathrm{t} 1$ seconds in order to monitor the route setup sate, wherein $\mathrm{t} 1$ value is set according to the node mobility.

If the intermediate node is under serious low battery state or the received signal is very weak, then the intermediate node will establish a HLP group with TTL setting as 1 and then send it to the neighbor node thereof. After receiving HLP data packet, the neighbor node will check the route availability in the routing list of the destination node thereof according to HLP data packet description. If the route is available, then the neighbor node will return the routing information to the downstream node in HLP data packet sent by the above intermediate node. After receiving the routing information, the downstream node will update the routing list. The data packet will be transmitted in the new route in order to avoid the data packet loss caused by link interruption.

If the node under low battery state is the destination node, then the node will send the instruction for transmission suspension to the source node in order to avoid possible data packet loss and resource wastage. Meanwhile, it is necessary to check whether the port queue length exceeds QThresh in order to avoid congestion.

If the alternate single-hop node route has not yet been found after the timer expires, namely timeout, then the node concerned will send RCR (Route Change Request) to the source node. After receiving RCR, the source node will execute the route discovery process for the destination node in order to find an alternate route before the link interruption of current route. Meanwhile, the routing list of the neighbor node will be updated along with the route change. If the node of which the total energy will be lost is the source node, then the node will continue the data transmission till the total energy loss process is ended.

If there is an alternate route leading to the destination node during the local route repair process, this mechanism will rapidly adapt to network change, thus to not only improve the data packet delivery ratio, but also reduce the quantity of packet losses and the occurrence of time delay. Furthermore, the data packet will not be transmitted to any unavailable intermediate node, thus to reduce resource wastage. 


\section{Algorithm 2. Route Maintenance}

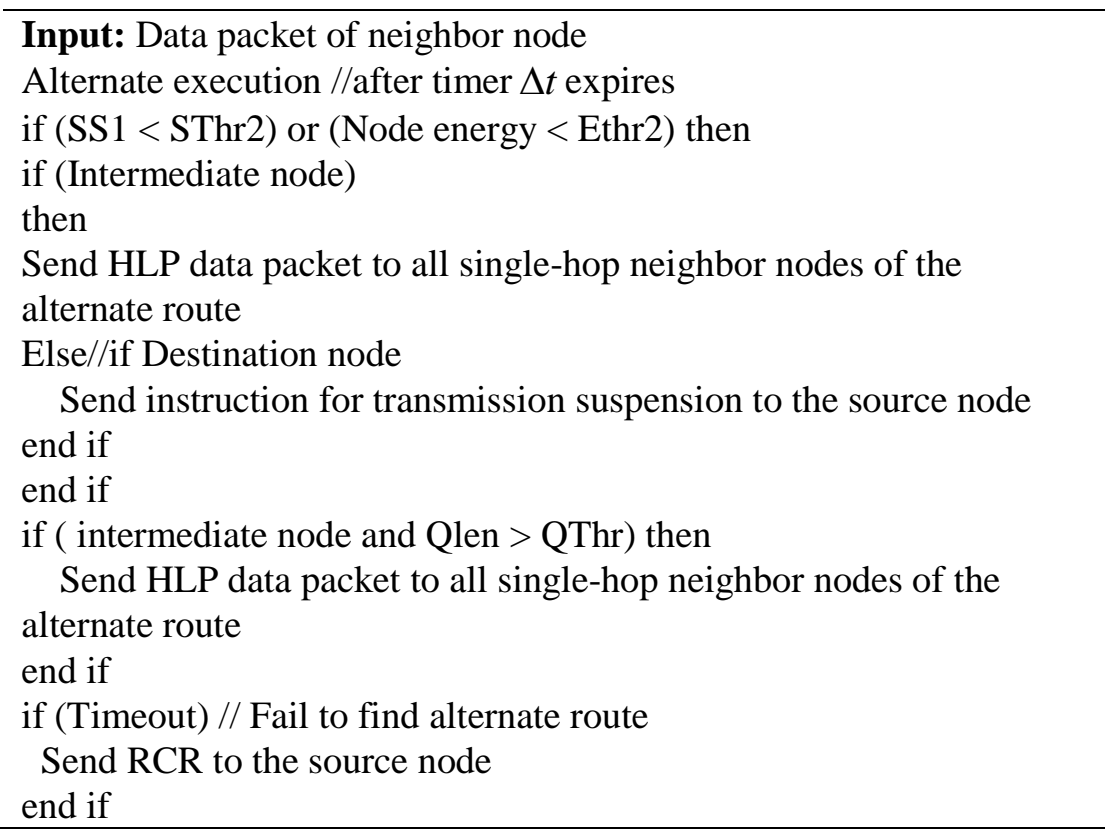

\section{Experiment Result and Analysis}

\subsection{Performance Index}

NCO (Normalized Control Overhead) refers to the specific value of the control packet transmitted thereby and the control packet received by the destination node.

PDR (Packet Delivery Ratio) refers to the specific value of the quantity of the data packets received by the destination node and the quantity of the data packets sent by the source node.

Node rest energy variance: this index is adopted to calculate the node energy use distribution.

End-to-end delay: this index is used to evaluate the mean time for transmitting the data packet to the destination.

\subsection{Simulation Parameters}

The simulation experiment is carried out in simulation tool NS2 [12], and the simulation environment parameters are as shown in Table 1. Specifically, the number of nodes is 50 , the topological size is $1000 \mathrm{~m} \times 500 \mathrm{~m}$, the flow number is 10 , the transmission range of the node is $250 \mathrm{~m}$ and the size of each data packet is 512 bytes.

Table 1. Simulation Parameters

\begin{tabular}{cc}
\hline Parameter Name & Value \\
\hline Movement Model & Random waypoint \\
Delivery Ratio (Packets/second) & 10 \\
Node Speed & $0, \quad 5, \quad 10, \quad 15, \quad 20$ \\
P1, P2 & 0.5 \\
\hline
\end{tabular}




\begin{tabular}{cc}
\hline Delivery Ratio (Packets/second) & 10 \\
SThr1 & $1.5 \times \mathrm{RxThr}$ \\
SThr2 & $1.2 \times \mathrm{RxThr}$ \\
Ethr1 & $10 \%$ of battery capacity \\
Ethr2 & $5 \%$ of battery capacity \\
QThr & $80 \%$ of port queue length \\
\hline
\end{tabular}

\subsection{Simulation Result Analysis}

In allusion to different movement speeds, namely: 0, 5, 15 and 20, a group of wide simulations is carried out. After 10 simulations, the mean values of the obtained results are taken as the performance index values.

Figure 1 shows that NCO caused by link interruption and route repair is increased along with the increase of mobility. Under low mobility, NCO values of the proposed algorithm, RSEA and LAER are respectively 5.1\%, 5.4\% and 6.3\%. Under high mobility, NCO values of the proposed algorithm, RSEA and LAER are respectively $22.8 \%, 26.2 \%$ and $31.3 \%$. RSEA shows better performance in highspeed dynamic environment. Due to the adoption of "Make-before-Break" mechanism and the local route repair mechanism, compared with other two routing protocols, the proposed routing protocol has less control overhead and can more early predict link interruption so as to switch the original route to the alternate route for continuous data transmission before link interruption.

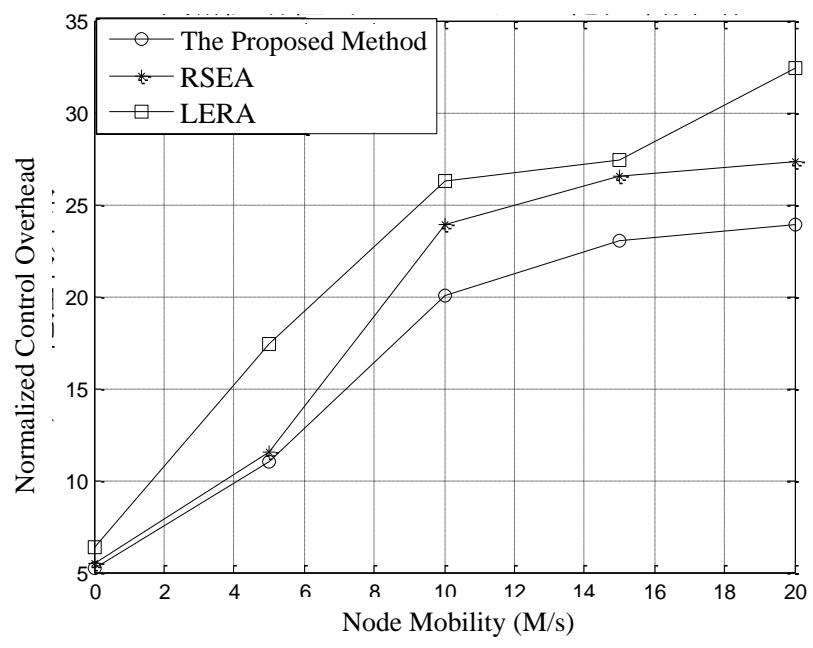

Figure 1. Relation between Control Overhead and Node Mobility

According to Figure 2, PDR values of the three protocols are reduced along with the increase of speed, and PDR reduction reason lies in frequent link interruptions and new routing operations when the speed is increased. Under high mobility, PDR values of the proposed method, RSEA protocol and LAER protocol are respectively 94.7\%, 93.8\% and 91. 3\%. PDR value of the propose method is about $1 \%$ higher than that of RSEA, because the propose method has "Make-before-Break" route maintenance mechanism and can significantly reduce the quantity of data packet losses caused by unpredictable link interruption. 


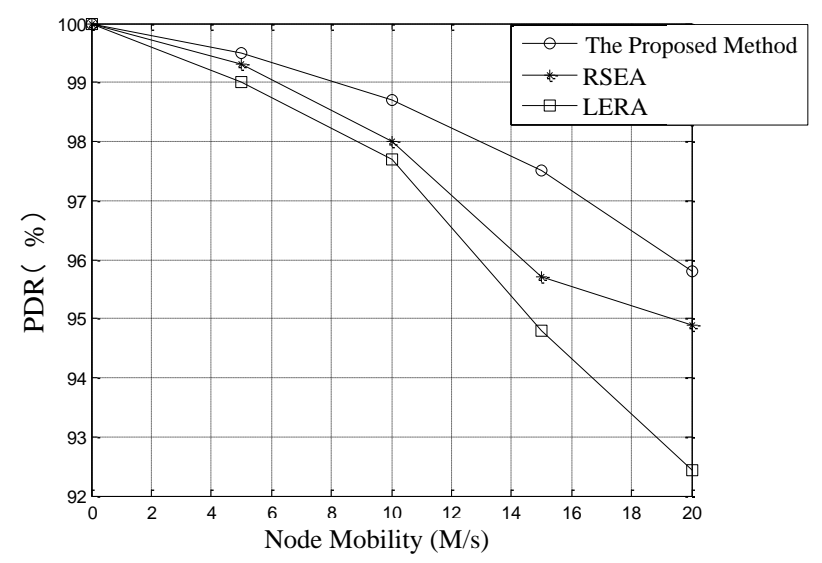

Figure 2. Relation between PDR and Node Mobility

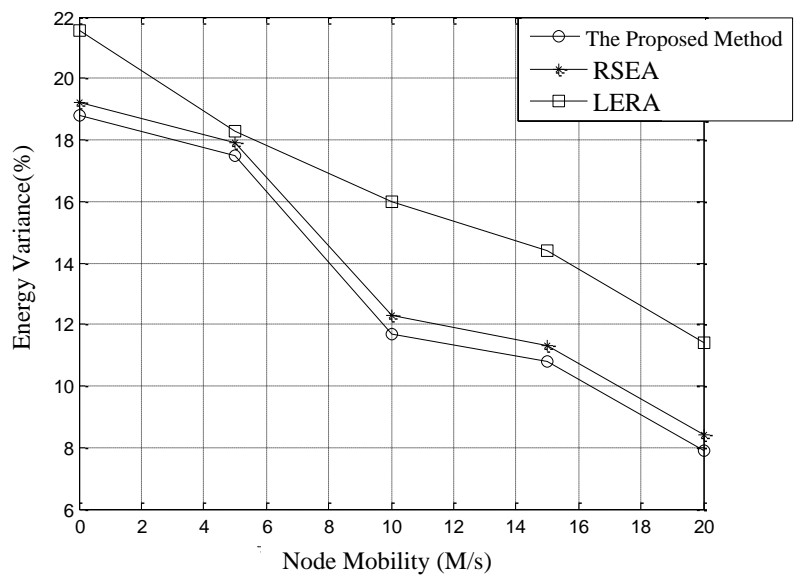

Figure 3. The Relationship between Energy Variance and Node Mobility

The variance of rest node energy is as shown in Figure 3, and this parameter represents the load balancing capability of the routing protocol. According to the observation, due to frequent route switching operations, the variance of the rest node energy is gradually reduced along with the increase of the mobility. Under low mobility, the energy variance percentages of the proposed method, RSEA and LAER are respectively $18.8 \%, 19.2 \%$ and $21.54 \%$. Under high mobility, the energy variance percentages of the proposed method, RSEA and LAER are respectively $7.8 \%, 8.3 \%$ and $11.3 \%$.

According to Figure 4, the end-to-end delays of the three protocols are increased along with the increase of mobility, because of frequent route interruptions. Under high mobility, the end-to-end delays of the proposed method, RSEA and LAER are respectively $0.18 \mathrm{~s}, 0.21 \mathrm{~s}$ and $0.32 \mathrm{~s}$. Due to the capability of avoiding the link interruption caused by node mobility and battery energy loss, the proposed method has minimum delay. Additionally, RSEA protocol also has "Make-before-Break" route maintenance mechanism and can rapidly adapt to network change. Under high mobility, the performance of the proposed method is $14 \%$ higher than that of RSEA protocol. 


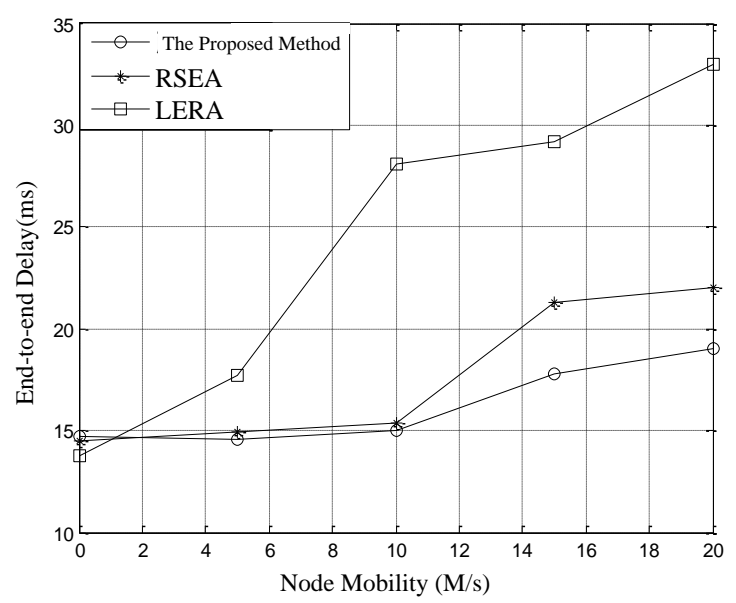

Figure 4. The Relationship between the End Delay and Node Mobility

\section{Conclusion}

The specific steps of RSEA-RM routing protocol are mainly discussed in this paper and mainly include route discovery, routing and route maintenance. Relevant experiment is carried out in NS2 to compare this protocol with other similar routing protocols such as RSEA and LAER, and these routing protocols are repeatedly simulated and compared under several conditions. The experiment result shows that the proposed scheme can not only significantly improve data packet delivery ratio through early warning, but also reduce the control overhead and the delay caused by unpredictable link interruption. Compared with LAER, RSEA-RM routing protocol can reduce node energy variance and improve network partition time and has superior performance in high-speed dynamic network, but the delay of the improved method is slightly higher than that of LAER protocol under low mobility. In future, the proposed protocol will be researched under different node densities and flow rates and in different movement models.

\section{Acknowledgement}

The research is supported by China National Nature Science found ( 61103099), Zhejiang province Nature Science Found(Q14F020032), Hangzhou Science Technology Project(20140633B34) and Hangzhou Key Subjects Found(2013X1010)

\section{References}

[1] Bang Wang, (2011). Coverage problems in sensor networks: A survey. ACM Comput. Surv., 43(4):1-32.

[2] Kar S, (2013). Node placement for connected coverage in sensor networks. in Proceedings of the Workshop on Modeling and Optimization in Mobile, Ad-Hoc and Wireless Networks. France: Sophia Antipolis, 41-48.

[3] Misra, S, Venkata Krishna, P., Saritha V, (2012). LACAV: an energy-efficient channel assignment mechanism for vehicular ad hoc networks. J.Supercomput., 62(3):1241-1262.

[4] Saipulla A, Cui J H, (2014). Barrier coverage: Foundations and design[J].In The Art of Wireless Sensor Networks, 45(7):59-115.

[5] Selina S, Fernaz N, (2015). Network Lifetime Aware Area Coverage for Clustered Directional Sensor Networks. IEEE Transactions on Computers, 23(4):1-9.

[6] Tian D, (2012). Georganas. A coverage-preserving node scheduling scheme for large wireless sensor networks .Proceedings of the 1st ACM International Workshop on Wireless Sensor Networks and Applications, ser. 32-41.

[7] Yi Wang and Guohong Cao, (2011). Barrier coverage in camera sensor networks. In Proceedings of the Twelfth ACM International Symposium on Mobile Ad Hoc Networking and Computing,12(3):1-10. 
[8] Yuanyuan Zeng, Naixue Xiong (2010). Connectivity and coverage maintenance in wireless sensor networks.J Supercomput,52(8):23-46.

[9] Zhihan Lv, Chantal Esteve, Javier Chirivella and Pablo Gagliardo. Clinical Feedback and Technology Selection of Game Based Dysphonic Rehabilitation Tool. 2015 9th International Conference on Pervasive Computing Technologies for Healthcare (PervasiveHealth2015), IEEE, 2015.

[10] Jiachen Yang et al. A Low-Power and Portable Biomedical Device for Respiratory Monitoring with a Stable Power Source. Sensors 15.8 (2015): 19618-19632.

[11] Zhanwei Chen, Wei Huang, and Zhihan Lv. Towards a face recognition method based on uncorrelated discriminant sparse preserving projection. Multimedia Tools and Applications (2015): 1-15.

[12] Xiaoming Li, et al. XEarth: A 3D GIS Platform for managing massive city information. Computational Intelligence and Virtual Environments for Measurement Systems and Applications (CIVEMSA), 2015 IEEE International Conference on. IEEE, 2015.

[13] Zhihan Lv, et al. A framework for multi-dimensional WebGIS based interactive online virtual community. Advanced Science Letters 7.1 (2012): 215-219.

[14] Zhihan Lu, Muhammad Sikandar Lal Khan, and Shafiq Ur Réhman. Hand and foot gesture interaction for handheld devices. Proceedings of the 21 st ACM international conference on Multimedia. ACM, 2013.

[15] Zhihan Lv, et al. Finger in air: Touch-less interaction on smartphone. Proceedings of the 12th International Conference on Mobile and Ubiquitous Multimedia. ACM, 2013.

[16] Xiaoming $\mathrm{Li}$, et al. Traffic management and forecasting system based on 3d gis. International Symposium on Cluster, Cloud and Grid Computing (CCGrid). IEEE. 2015.

[17] Zhang, Xu. Spike-based indirect training of a spiking neural network-controlled virtual insect. 2013 IEEE 52nd Annual Conference on Decision and Control (CDC). IEEE, (2013)

[18] Wei Ou, Zhihan Lv, Zanfu Xie. Spatially Regularized Latent topic Model for Simultaneous object discovery and segmentation. The 2015 IEEE International Conference on Systems, Man, and Cybernetics (SMC2015).

[19] Wang, Ke. "Using Simulation to Explore Distributed Key-Value Stores for Exascale System Services." 2nd Greater Chicago Area System Research Workshop (GCASR). (2013).

[20] Yi Wang, Yu Su, Gagan Agrawal. A Novel Approach for Approximate Aggregations Over Arrays. In Proceedings of the 27th international conference on scientific and statistical database management, ACM, (2015).

[21] Zhihan Lv, Alaa Halawani, Shengzhong Feng, Shafiq ur Rehman, Haibo Li. Touch-less Interactive Augmented Reality Game on Vision Based Wearable Device. Personal and Ubiquitous Computing. (2015).

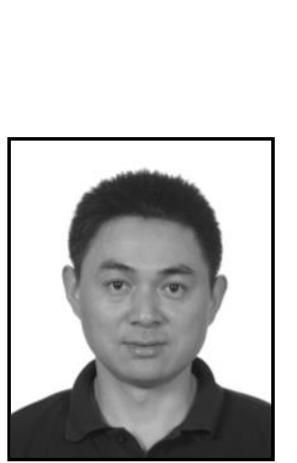

\section{Authors}

Shihua Cao, He was born in 1972. He received his Bachelor degree in Computer Science and Technology from Hangzhou Teacher's College, China in 2005, his MSE from Beijing University of Posts Telecommunications, China in 2007. He is currently an associated professor and Senior Engineer in Qianjiang College of Hangzhou Normal University. His research area is mainly in the area of Wireless Sensor Network, Energy Harvesting, the Internet of Things. He has published. 
International Journal of Grid and Distributed Computing

Vol. 9, No. 8 (2016) 